\title{
Research on Automated Warehouse Scheduling System Based on Double Label and Sorting Algorithm
}

\author{
Yang Xiao ${ }^{1, a}$, Man Yun-chao ${ }^{1}, \mathrm{Xu} \mathrm{Jian}^{1}$, Bai Shan ${ }^{1}$, and Liang Jiao ${ }^{1}$ \\ ${ }^{1}$ RIAMB (Beijing) Technology Development Co., Ltd. Beijing 100120, China
}

\begin{abstract}
Through the research on the RFID's User tag memory and its coding mode, a double-label management mode based on the combination of RFID tag and barcode is proposed, which realizes the binding of information in the warehouse logistics process, the convenient transmission and traceability between the upstream and downstream departments. The interface between the scheduling system and the equipment control system is designed, and the scheduling algorithm based on priority ranking is put forward to realize material outbound in order in warehouse logistics system. This method is an extension of the warehouse and logistics information system, which can solve the problem of the accuracy of the material outbound in sequence to the downstream departments.
\end{abstract}

\section{Introduction}

Enterprises usually introduce Automatic Storage \& Retrieval System (ASRS) to realize the scientific management of production logistics information. ASRS generally includes stereoscopic warehouse, stacker, conveying equipment, logistics control system and logistics information system, among which the logistics information system includes warehouse management system (WMS) and warehouse scheduling system (WCS). The management of product logistics information is simple in the conventional WMS and WCS, which only needs to complete the function of automatic allocation of the cargo space address, bookkeeping and write-off according to the inbound and outbound lists provided by ERP.

With the improvement of production technology and the expansion of production scale, the conventional WMS and WCS has been unable to adapt to the more complex management requirements and more diverse use cases. Therefore, the research on WMS and WCS is also deepening step by step. For example, the model of library selection path is analysed by Genetic Algorithm [1], the optimization of cargo location allocation is realized by combining the advantages of Polychromatic Sets algorithm, Particle Swarm Optimization algorithm and Simulated Annealing algorithm [2], a meta-heuristic algorithm based on Simulated Annealing optimizes the access process [3].

The previous WMS adopts the single-label accounting mode of only pallet label or only material label. Once the material is incorrectly bound to the pallet, the system cannot verify the material information. The previous WCS adopts the inbound mode of empty pallet and material off-line binding, and the outbound mode of batch random disorderly according to the outbound lists, which cannot solve the requirement of strict production sequence for the downstream department.

In this paper, a double-label management mode based on the combination of RFID and barcode is proposed to ensure the accuracy and traceability of material information, a new scheduling system based on sorting algorithm is introduced to solve the business requirements of outbound sequentially strictly.

\section{Development background and demand analysis}

\subsection{Development background}

Figure 1 shows the layout of one roadway in this project, mainly including shelves, stacker, shuttle vehicles (RGV), external access equipment and manual intervention area.

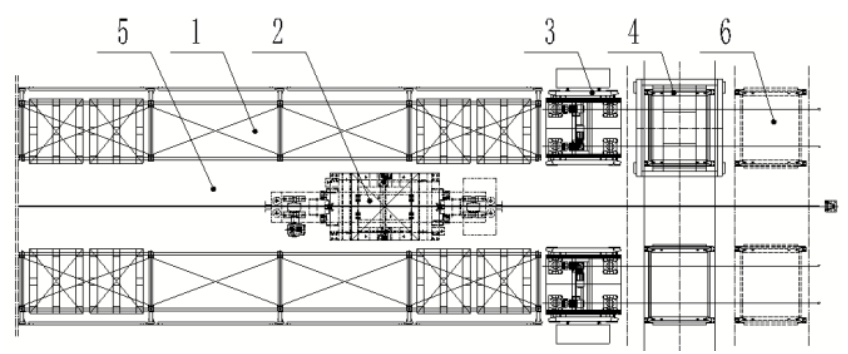

(1) Shelves (2) Stacker (3) RGV (4) External access equipment (5) Railway (6) Manual intervention area

Figure 1. Project layout

There are about two rows of shelves in each roadway, with multiple layers and multiple columns. Each cargo

\footnotetext{
a Corresponding author: 54454639@qq.com
} 
space can store a pallet. The stacker is the equipment to complete the storage task in the warehouse, and interacts with the RGV to realize the inbound of materials. RGV is responsible for transporting the materials from external access equipment to the stacker inbound, and transporting the materials from the stacker to the external access equipment outbound. WMS and WCS are responsible for real-time monitoring, scheduling, management and maintenance of warehouse logistics information.

\subsection{Demand analysis}

The requirements of this type of project have the following characteristics:

(1) A class of non-standard pallets, empty pallets and empty pallets cannot be stacked for storage;

(2) The empty pallets are stored in the warehouse, and when necessary, they will be outbound to be bind to the materials' information;

(3) Material information can be transferred easily and tracked reliably among different departments;

(4) The downstream departments have strict requirements on the production sequence and need to realize the cross outbound of materials of different specifications.

For the above requirements, this paper will provide solutions in the following sections.

\section{RFID technology and double-label management mode}

\subsection{RFID technology}

RFID, radio frequency identification technology, is a technology that realizes contactless information transmission by using spatial coupling of radio frequency signals. The system of RFID is generally composed of electronic tags, readers and antennas, data transmission and processing systems, etc. [4].

According to the relevant standards, the working frequency of the RFID system has many ranges. As a universal frequency band, the uhf RFID system between 860 and $960 \mathrm{MHz}$ has attracted the most attention and developed the fastest [5]. Uhf RFID technology has many advantages, such as large read-write distance, wide application, fast transmission speed, allowing multi-label batch reading and so on. Currently, RFID system has a relatively complete coding system including EPC Global and Ubiquitous ID, etc. Currently, most uhf systems use the former, which conforms to EPC Gen2 protocol. Such systems are divided into four independent storage blocks: Reserved area, EPC area, TID area and User area. RFID technology is widely used in practice [6-8].

\subsection{Double-label management mode}

Double label management mode refers to: the pallet has the barcode label, the material has the RFID tag, the two types of label will need to be bound inbound and checked outbound. Figure 2 shows the diagrammatic drawing of the double label and its code reader.

Barcode label do not has reproducible function, it can read through the barcode reader, each pallet of the barcode is not the same, barcode label only circulate within the system. RFID reader can read and write RFID tags, using tag encryption to prevent arbitrary modification of data, to ensure data security. When inbound, according to the coding rules by the ERP, the User area of the RFID tag on the material will be written into the data and sent back to the ERP. The User area can store 64 bytes of data in hexadecimal format.

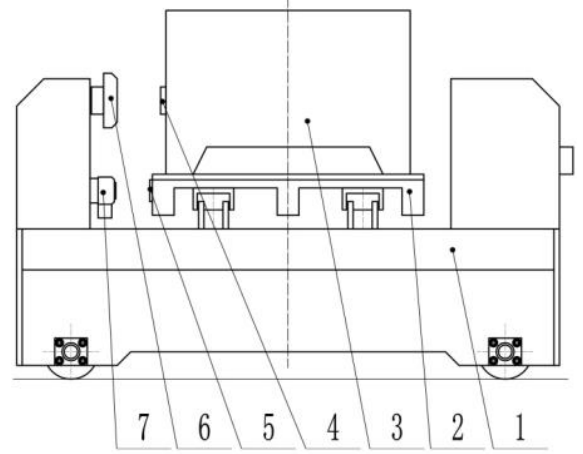

(1) RGV (2) Pallet (3) Material (4) RFID tag (5) Barcode tag (6) RFID reader (antenna) (7) Bar- code reader

Figure 2. The diagrammatic drawing of the double label and its code reader

As shown in table 1, the coding rule of the label can contain multiple fields, such as manufacturing shop, stock number, production number, grade, characteristic, inspection number and other production flow of information. When the field information is insufficient, add 0 before it. When outbound, the material will transfer a variety of information to the downstream department through the RFID chip for its collection and processing.

Table 1. User area coding rules of RFID

\begin{tabular}{|c|c|c|}
\hline $\begin{array}{c}\text { Byte } \\
\text { offset }\end{array}$ & Field & Coded message \\
\hline $1 \sim 4$ & $\begin{array}{c}\text { Manufacturing } \\
\text { shop }\end{array}$ & 00003235 \\
\hline $5 \sim 20$ & Stock number & $\begin{array}{c}000000003232343533333537 \\
37323232\end{array}$ \\
\hline $21 \sim 52$ & Production number & $\begin{array}{c}000000000000323031393130 \\
313038383839393737373232 \\
3139373636353231\end{array}$ \\
\hline $53 \sim 56$ & Grade & 00414141 \\
\hline $57 \sim 60$ & Characteristic & 0037383434 \\
\hline $61 \sim 64$ & Inspection number & 00000031 \\
\hline
\end{tabular}

The double-label management mode inherits the convenient advantage of the conventional WMS that only manages the accounts according to the barcode of pallet, that is, the scheduling system collects the barcode of pallet from the equipment control layer, obtains the specific information of material from the relevant interface, binds the barcode and FRID tag and stores to the database. At the same time through RFID tags to make up for the past cannot carry out inventory of goods. 
That is, if the information source is wrong, the binding relationship is wrong and cannot be detected until the manual recheck. The double-label management mode realizes the double-dimensional insurance of the data in this link. The two labels compare with each other and correct each other. Once the material information is found to be wrong, the system can timely correct the error by means of re-binding to ensure the accuracy of the material information.

This scheme basically solves the problem that the storage information is inconsistent with the real goods in some cases, and provides a strong and accurate guarantee for the downstream production. At the same time, it also saves the labour cost and time cost of subsequent error correction after the previous information error, so as to make the whole system run more efficiently.

\section{Design of scheduling system}

\subsection{Interface and class design with equipment automation control system}

The interface design of scheduling system and control system needs to be based on the simple and clear design idea of the interaction information, which includes collecting the device status and issuing the task. The scheduling system issues the task to the stacker, such as inbound, outbound, check storage, the stacker needs to return the operation data at the same time. The inbound binding and outbound verification processes of the double-label mode are completed on RGV. The interface protocol between the scheduling system and RGV is designed (Table 2), and RGV is defined as an object.

Table 2. Interface protocol of scheduling system and RGV

\begin{tabular}{|c|c|}
\hline RGV To WCS & WCS To RGV \\
\hline Request code & Response code \\
\hline Barcode of pallet & Barcode of pallet \\
\hline RFID code of material & RFID code of material \\
\hline Allowed to put goods on RGV & $\begin{array}{c}\text { Types of outbound (empty } \\
\text { pallet, pallet + material) }\end{array}$ \\
\hline
\end{tabular}

In addition to the fields defined in the interface protocol as member variables, the member methods to be defined include: empty pallet inbound interaction, empty pallet and material binding interaction, material outbound sequence verification interaction, material unloading sequence verification interaction, material after-unloading verification interaction, and ERP interface interaction, etc.

\subsection{Design of in-bound process}

Figure 3 is programming flowchart of inbound process. In general, this kind of non-standard empty pallets needs to be stored in warehouse first, and then according to the order of material inbound list, empty pallets need to be outbound and then bound to the material. In consideration of the more convenient interaction between the control system and the code reader, the control system is responsible for triggering the code reader to read the label data and then upload the data to the scheduling system after sorting it out.

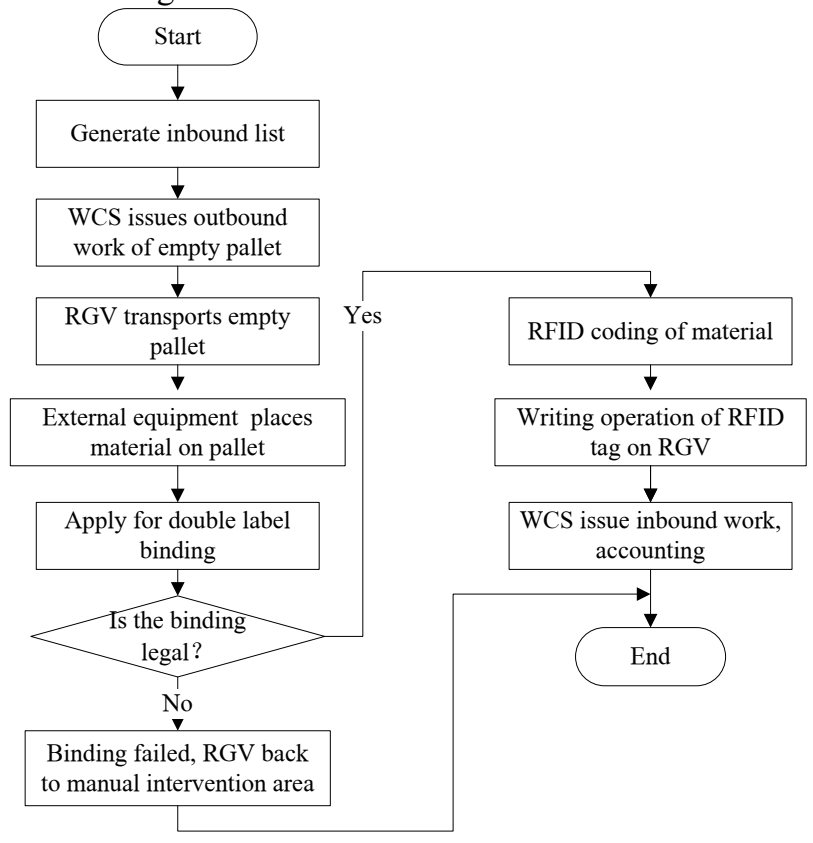

Figure 3. Programming flowchart of inbound process

According to different business process, the scheduling system issues instructions to the control system to complete the write operation of RFID tags, the inbound binding process and the outbound verification process. Another way is to process RFID tag through handheld terminals, which is generally used for manual processing and has the characteristics of convenient and flexible operation.

\subsection{Design of out-bound process}

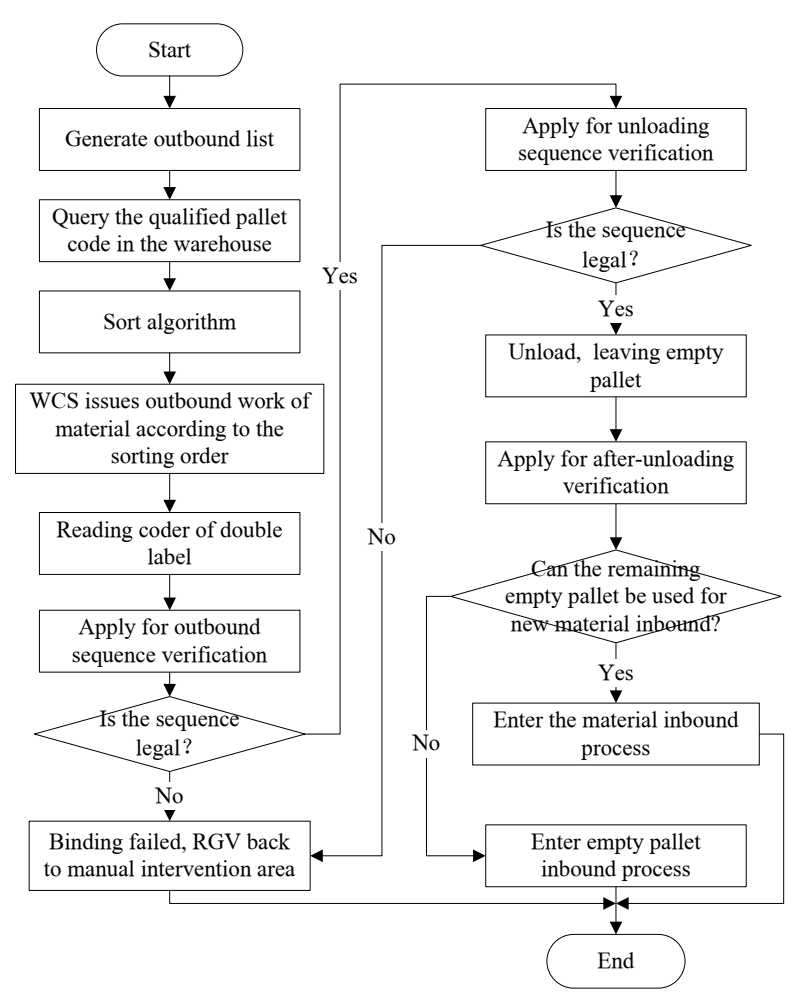

Figure 4. Programming flowchart of outbound process 
Figure 4 is programming flowchart of outbound process. Notes that the scheduling system needs to determine whether the remaining empty pallets after unloading can be directly used in the new round of material inbound process according to the current working conditions, so as to reduce unnecessary logistics handling process.

Usually, the database needs to design the work record table, inventory flow table, current work task list for recording the current or previous work type and work status, which includes the work type of inbound, outbound, inventory, etc., the work status includes waiting for release, in the process of execution, completed, no pallet for picking up, double inbound, etc. However, the fields in the above table are relatively rich, which is inconvenient to verify the outbound sequence of materials. Therefore, the design of the outbound sequence table is shown in table 3 . The sequence of data in the table is the outbound sequence of materials.

Table 3. Field description of outbound sequence table

\begin{tabular}{|c|c|c|c|c|}
\hline Field & Description & $\begin{array}{c}\text { Data } \\
\text { type }\end{array}$ & Length & null \\
\hline ID & Primary key & BigInt & & isnull \\
\hline PALLETID & Pallet barcode & VarChar & 50 & \\
\hline RFID & RFID tag & VarChar & 100 & \\
\hline OUTSEQ & $\begin{array}{c}\text { Outbound } \\
\text { sequence } \\
\text { verification }\end{array}$ & TinyInt & & \\
\hline ULSEQ & $\begin{array}{c}\text { Unloading } \\
\text { sequence } \\
\text { verification }\end{array}$ & TinyInt & & \\
\hline AFULSEQ & $\begin{array}{c}\text { After } \\
\text { unloading } \\
\text { verification }\end{array}$ & TinyInt & & \\
\hline
\end{tabular}

Table 4. Interaction protocol of scheduling system and RGV description

\begin{tabular}{|c|c|c|c|}
\hline \multicolumn{2}{|c|}{ RGV TO WCS } & \multicolumn{2}{c|}{ WCS TO RGV } \\
\hline $\begin{array}{c}\text { Req- } \\
1\end{array}$ & $\begin{array}{c}\text { Description } \\
\text { pallet and material } \\
\text { binding verification }\end{array}$ & $\begin{array}{c}\text { Resp- } \\
\text { onse }\end{array}$ & Description \\
\hline 2 & $\begin{array}{c}\text { Apply for outbound } \\
\text { sequence verification }\end{array}$ & 2 & $\begin{array}{c}\text { Empty pallet and } \\
\text { material binding } \\
\text { is legal }\end{array}$ \\
\hline 3 & $\begin{array}{c}\text { Apply for unloading } \\
\text { sequence verification } \\
\text { sequence } \\
\text { verification is } \\
\text { legal }\end{array}$ \\
\hline 4 & $\begin{array}{c}\text { Apply for after- } \\
\text { unloading verification }\end{array}$ & 4 & $\begin{array}{c}\text { Unloading } \\
\text { sequence } \\
\text { verification is } \\
\text { legal }\end{array}$ \\
\hline & & $\begin{array}{c}\text { After-unloading } \\
\text { enter the material } \\
\text { inbound process }\end{array}$ \\
\hline
\end{tabular}

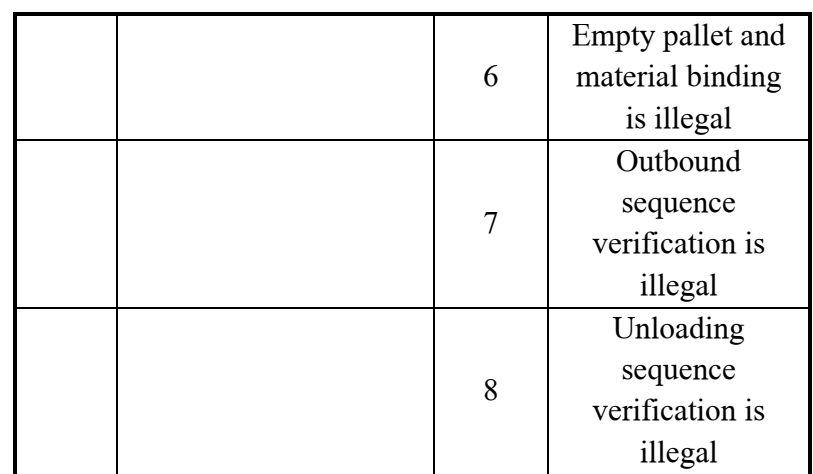

As shown in table 4, the scheduling system needs to verify the pallet and materials for each time according to the outbound sequence table and the pallet barcode, RFID tag and request code uploaded by RGV. If the verification is legal, the corresponding response code is issued to RGV and the corresponding fields in the outbound sequence table are updated. If the validation illegal, the corresponding fields in the outbound sequence table are not processed to ensure that the materials are verified in strict sequence. Add a manual processing mode, when the verification is illegal, manual to the corresponding field of the outbound sequence table to update the completion flag.

\subsection{Design of out-bound sorting algorithm}

\subsubsection{Sorting Algorithm}

The outbound lists issued by the ERP system include materials of various grades or characteristics, and shall be according to such as $a$ pallet of grade $A$ material, $b$ pallet of grade $B$ material, $c$ pallet of grade $C$ material... $h$ pallet of grade $H$ material, once more $a$ pallet of grade $A$ material, $b$ pallet of grade $B$ material, $c$ pallet of grade $C$ material $\ldots h$ pallet of grade $H$ material, so the outbound sequence of such a cycle for use by the downstream department. After the scheduling system receives the outbound lists, it queries the material information in accordance with the principle of "first in, first out" in the database and inserts them into the outbound sequence table. However, the current outbound sequence table cannot meet the requirements of outbound in the circular order.

In this paper, a sorting algorithm based on priority is proposed and its main idea is to add a field in the outbound sequence table: priority. After the outbound sequence table is traversed once, the priority of each data is assigned with different priority values. At this time, the ascending order of the priority value in the outbound sequence table is the outbound sequence.

With $N a * a$ pallet of grade $A$ material, $N b^{*} b$ pallet of grade $B$ material, $N c^{*} c$ pallet of grade $C$ material... $N h^{*} h$ pallet of grade $H$ material is the sample, through a traversal to complete the sorting, in which $\mathrm{Na}, \mathrm{Nb}, \mathrm{Nc} \ldots \mathrm{Nh}$ is a natural number, as shown in the following steps. 
Step 1: add the field priority in the outbound sequence table: Fin

Step 2: define the total number of outbound pallets per round

$$
C y c=a+b+c+\ldots+h
$$

Step 3: define $A i, B i, C i \ldots H i$, the data type is int, where $H i$ represents that the data of the item $i$, grade $H$ material has been processed in the traversal of the outbound sequence table;

Step 4: when dealing with the data of the item $i$, grade $A, B, C \ldots H$, there is a formula

$$
\begin{aligned}
& \operatorname{Fin}(A i)=C y c *(\operatorname{int})((i-1) / a)+i \% a+0 \\
& \operatorname{Fin}(B i)=C y c *(\operatorname{int})((i-1) / b)+i \% b+a \\
& \operatorname{Fin}(C i)=C y c *(\operatorname{int})((i-1) / c)+i \% c+(a+b) \\
& \ldots \\
& \operatorname{Fin}(H i)=C y c *(\operatorname{int})((i-1) / h)+i \% h \\
& +(a+b+c+\ldots+g)
\end{aligned}
$$

Where ' $/$ ' is the integer division operator, ' $\%$ ' is the remainder operator, and ' int' is the forced negative integer operator.

For example, the first part of (2), $C y c *(\operatorname{int})((i-1) / h)$, represents the sorting reference priority value of different rounds. The second part, $i \% h$, represents the priority value of the same round and the same material. And the third part, $(a+b+c+\ldots+g)$, represents the specified order of different materials. Therefore, the smaller field Fin is, the higher priority is, and the earlier the outbound sequence is.

Step 5: sort the processed outbound sequence table in ascending order according to field Fin .

\subsubsection{Algorithm Complexity}

The processing of the conventional sorting algorithm for the outbound sequence table is: for the table of $n$ data table, $n-1$ times of traversal, traversing the data in accordance with the sorting requirements, and it is inserted into another table in the order of the completion of the traversal, the time complexity of the conventional sorting algorithm is $O\left(n^{2}\right)$. The sorting algorithm in this paper only needs to be traversed once, and the time complexity is $O(n)$.

\subsubsection{Algorithm Verification Experiment}

Table 5. Sorting algorithm processing before and after the outbound order table

\begin{tabular}{|c|c|c|}
\hline $\begin{array}{c}\text { The sequence number } \\
\text { in the table before } \\
\text { sorting }\end{array}$ & Grade & $\begin{array}{c}\text { After the sorting the } \\
\text { field Fin }\end{array}$ \\
\hline 1 & C & 3 \\
\hline 2 & A & 1 \\
\hline 3 & B & 2 \\
\hline 4 & B & 6 \\
\hline 5 & A & 0 \\
\hline
\end{tabular}

\begin{tabular}{|c|c|c|}
\hline 6 & A & 5 \\
\hline 7 & C & 7 \\
\hline 8 & A & 4 \\
\hline 9 & A & 9 \\
\hline 10 & C & 11 \\
\hline 11 & A & 8 \\
\hline 12 & B & 10 \\
\hline
\end{tabular}

In this experiment, 2 pallets of grade $A$ material, 1 pallet of grade $B$ material and 1 pallet of grade material $C$ should be outbound in sequence. The outbound sequence table before and after the sorting algorithm is shown in table 5. Before sorting algorithm, the order of materials in the outbound order table is only the result selected in the material table, which does not meet the outbound order of materials. After the sorting algorithm, the material can be outbound strictly in sequence.

\section{Conclusion}

In this paper, a double-label management mode based on RFID and barcode combination is proposed, and WCS with material sorting requirements is studied to realize the convenient transmission and reliable traceability of material information between upstream and downstream logistics departments. A sorting algorithm based on priority, which solves the problem of sorting materials by group and grade outbound, has been applied to multiple projects.

Given the limited space available, this paper does not discuss the double-label management mode and its application in detail, the research in this field has been widely developed recently and is worth further study in the future. With the increasingly wide application of ASRS technology, the usage scenarios are becoming more and more complex, in order to solve the emerging needs, the research of WMS and WCS is also gradually in-depth, scheduling algorithm will also be more abundant, efficient, flexible and diverse, so as to make the logistics warehousing system more adaptable.

\section{References}

1. Xia X.K, "Optimization Model and Algorithm Design of Warehouse Operation Based on Large Data," M.S. thesis, Lanzhou Jiaotong University, Lanzhou, China, 2017.

2. Yang Yi. "Optimization of Location Assignment in AS/RS," Modern Manufacturing Engineering, vol. 12, pp. 134-141, 2014.

3. Massimo Bertolini, et al. " Optimizing Retrieving Performance of an Automated Warehouse for Unconventional Stock Keeping Units," Elsevier B.V, vol. 39,2019,.

4. Tan Min, Liu Yu, Zen J.F, RFID Technology System Engineering and Application Guide. Beijing: Electronic Industry Press, 2007.

5. Zhang L.J. " Development of radio frequency identification technology," Mechanical Management and Development, vol. 24, pp. 89-90, 2009. 
6. Gligorije Mirkov " RFID technology in the function of generating flexible robotic sequences of the FMC," Springer Berlin Heidelberg, vol.41(12), 2019.

7. WANG W.J. " Digital Logistics Warehouse Management System Based on Radio Frequency Identification Technology," Science Technology and Engineering, vol.19(02), 2019.

8. ZHAO D.D, YANG J.D, et al. " Research on digital logistics warehouse inspection based on rfid technology," Automation \& Instrumentation, vol.04, pp. 108-111, 2020. 